\title{
Sesgos en la Estimación de Probabilidades para Dos Situaciones Secuenciales Aleatorias ${ }^{1}$
}

\section{Traits in the Estimate of Probabilities for Two Random Sequential Situations}

\author{
Horacio Félix Attorresi², Alcira Myriam García Díaz y Héctor Omar Pralong \\ Instituto de Investigaciones de la Facultad de Psicología de la Universidad de Buenos Aires, \\ Argentina
}

(Recepción: Febrero 2008 - Aceptación: Mayo 2008)

\begin{abstract}
Resumen
Se analizaron secuencias aleatorias donde se debió tener en cuenta la estabilidad de las frecuencias relativas. Se puso en evidencia el heurístico de representatividad. Se utilizaron dos planteos de situaciones abiertas, donde se solicitó al entrevistado que realizara una estimación de probabilidades y que justificara su respuesta. En el primero, dicha indagación se efectuó al comparar proporciones de dos muestras de diferente tamaño, en el segundo, esta noción se expresó a través de la estabilidad de las frecuencias relativas en series de repeticiones de experiencias aleatorias. Se administraron los reactivos a doscientos siete estudiantes ingresantes a la Universidad. Las respuestas se categorizaron teniendo en cuenta la comprensión cognitiva alcanzada. Además se realizó un agrupamiento posterior de las mismas, en Correctas e Incorrectas para ambos reactivos, encontrándose asociación entre ellos.
\end{abstract}

Palabras claves: Probabilidad, Aleatoriedad, Sesgos.

\begin{abstract}
Random sequences, where stability in the relative frequencies had to be taken into account, were analyzed. The heuristics of representation was proved. Two different examples of open situations, where each interviewee was asked to make an estimate of probabilities and a justification for his answer were presented. In the first one, the enquiry was proved when comparing proportions of two samples of different size; in the second situation this notion expressed through the stability of the relative frequencies in series of repetitions of random experiences. Two hundred and seven students being admitted to university were given the devices. Their answers were categorized taking into account the level of cognitive comprehension achieved. The answers were grouped in different categories Correct and Incorrect for both devices as well, and a connection between them was found.
\end{abstract}

Key words: Probability, Random, Traits

1 Reconocimientos: La investigación que se presenta en este artículo fue realizada con subsidios de la Universidad de Buenos Aires (UBACyT P020 y P027) y de la Agencia Nacional de Promoción Científica y Tecnológica (ANPCyT PICT $2004 \mathrm{~N}^{\circ}$ 20909).

2 Correspondencia a: Horacio Félix Attorresi. Dirección Postal: Rivera Indarte $132-1^{\circ}$ A (1406) Buenos Aires, Argentina. Teléfono: (5411) 4637-0923 E-mail: horacioattorresi@fibertel.com.ar hattorre@psi.uba.ar 
En el área del razonamiento probabilístico es frecuente destacar la importancia de la formación en probabilidades y estadística, dada la incidencia que tienen estos conocimientos en la comprensión de situaciones basadas en la incertidumbre y que atraviesan distintos campos cognitivos. En el ámbito científico se han priorizado los enfoques deterministas y las explicaciones en torno de causas y efectos.

Existen antecedentes en investigaciones sobre aprendizaje de nociones probabilísticas en la enseñanza secundaria vinculadas a situaciones en las que interviene el azar, en las que se estiman probabilidades, encontrándose dificultades para alcanzar un aprendizaje significativo en temas de interpretación, estimación y cálculo de probabilidades de sucesos (Batanero, 2000; Konold, 1991; Lecoutre, 1985 y 1992; Lecoutre \& Cordier, 1990). Se advierte la utilización de estrategias de resolución no formales, basadas en un conocimiento cotidiano, en yuxtaposición con estrategias formales, coexistencia que puede entorpecer la comprensión acerca de lo incierto (Borovcnik \& Bentz, 1991; Serrano, Batanero, Ortiz \& Cañizares, 1998).

Carmen Díaz (2003) señala la existencia de trabajos de investigación en los que se estudia el efecto de la instrucción sobre los heurísticos y sesgos en el razonamiento probabilístico en estudiantes de Magisterio, encontrando que la instrucción en probabilidad no alcanza para eliminar nociones erróneas. Apoyándose en Konold (1989), la autora destaca que las nociones intuitivas acerca de la incertidumbre que las personas se forman a partir de su experiencia en la vida cotidiana no son suficientemente confrontadas en ámbitos educativos. También advierte, dada la población estudiada, el perjuicio que puede ocasionar la transmisión de estos errores en procesos posteriores de enseñanza.

Barragués, Guisasola \& Morais (2005) sostienen que hay escasos estudios acerca de cómo se entiende el azar y la probabilidad en estudiantes universitarios y señalan la conveniencia de estudiar cómo inciden las creencias y aprendizajes previos de los estudiantes sobre temas vinculados a estas nociones, así como los criterios que emplean para decidir sobre la verosimilitud de sucesos. En alumnos universitarios se ha encontrado un Sesgo Determinista que vincula causas y efectos a los fenómenos, que no les permite dar cuenta del carácter aleatorio de los mismos (Guisasola \& Barragués, 2002). Si el individuo descubre una razón lógica la pone en práctica rápidamente, desestimando incluso otras razones lógicas encontradas con anterioridad (Borovcnik \& Peard, 1996). Cardeñoso (2001) sostiene que el tipo de enseñanza puede afirmar dichas estrategias y no eliminar los sesgos que provoca. El lenguaje utilizado y el contexto en el cual se enmarcan los ejemplos y los reactivos, pueden constituirse en obstáculos para la construcción del conocimiento (Serradó, Cardeñoso \& Azcárate, 2005).

La realidad requiere de modelos probabilísticos para su interpretación, tanto como de la aritmética elemental. La probabilidad a través de sus aplicaciones ilustra cómo la matemática puede modelizar y resolver problemas reales.

La enseñanza de nociones probabilísticas puede llevarse a cabo mediante métodos heurísticos, a través del planteo de problemas concretos y la realización de experimentos reales o simulados.

Fischbein (1975) señala la carencia en el currículum escolar de situaciones en las que sea necesario constituir un pensamiento estadístico y probabilístico que amplíen el marco de las experiencias deterministas. En tal sentido este autor señala la conveniencia de desarrollar la intuición probabilística como una manera de caracterizar grados de incertidumbre que permitan comparar predicciones y extrapolaciones particulares con lo que realmente sucede. Aunque Piaget \& Inhelder (1951) plantean una postergación para la instrucción en nociones probabilísticas hasta que se halla constituido en los niños un pensamiento lógico o al menos nociones como la de proporcionalidad, Fischbein (1975) propone explorar intuiciones y precursores del conocimiento probabilístico buscando la existencia de conceptos de probabilidad parcialmente formados.

Nuestro conocimiento acerca de atributos y características, reunidos en un conjunto de individuos o más generalmente de elementos que forman un colectivo a estudiar (población), está 
basado en un número más o menos reducido de dichos elementos, parcialidad que se conoce como muestra. La Estadística a través de sus leyes indica el grado de confianza que se puede atribuir a las estimaciones basadas en una muestra, según el tamaño de la misma. Es sabido que la mejor estimación acerca de un conjunto de datos es alcanzada al considerar la media y ésta, a su vez, es más exacta cuando más datos hay. La denominada Ley de los grandes números asegura que la frecuencia de ocurrencia de un determinado evento aleatorio en una serie de $n$ ensayos (muestra) se estabiliza alrededor de un número llamado probabilidad, a medida que el tamaño de la muestra es mayor. Por otra parte, si se comparan las medias tomadas de muestras de un mismo tamaño n, se observa que existe variabilidad entre ellas. Si ahora repetimos la comparación entre medias obtenidas de muestras de un mismo tamaño m, siendo m menor que n encontraremos mayor variabilidad entre las medias de esta última serie, es decir, existe más inestabilidad entre las medias calculadas a partir de un número menor de datos. En síntesis, las medias muestrales presentan variabilidad, la cual aumenta al disminuir el tamaño muestral. Por esta razón, es más probable observar en muestras de menor tamaño, resultados más alejados de los esperados según la lógica probabilística.

Muchas características a estudiar (variables) son dicotómicas o dicotomizables, es decir que puede ocurrir un determinado suceso, denominado éxito, o no; en este último caso se dice que ocurre el fracaso. Estas situaciones simples pueden ser modelizadas a través de una variable aleatoria que sigue el modelo Bernoulli. Un ejemplo de esta situación podría ser registrar el sexo en un nacimiento. Suele ser de interés estudiar una variable que contabilice la cantidad de veces que ocurre el suceso de interés, el éxito, en una secuencia de situaciones de tipo Bernoulli, realizadas todas en las mismas condiciones. Esta variable contadora se dice que sigue una distribución Binomial y sus valores no suelen ser equiprobables, aun cuando la situación de Bernoulli que le da origen, sí pueda serlo. Un ejemplo de la variable binomial puede obtenerse al contabilizar la cantidad de varones que nacen en n nacimientos. Es decir, se define una variable Binomial tomando como base una variable Bernoulli, siendo un error muy común la confusión entre ambas, lo que lleva a su vez, a confundir la asignación de probabilidades a los valores de cada una de ellas.

Las personas suelen tener dificultades para tener en cuenta estos conceptos de la teoría estadística, entre otros, en particular las nociones sobre el tamaño muestral, por lo que muchas veces no pueden extraer inferencias correctas que los beneficiarían en su desempeño cotidiano. La ocurrencia de errores en el pensamiento y las conductas frente a la toma de decisiones en situaciones de incertidumbre que desestiman la lógica probabilística han sido estudiadas por diversos autores, entre otros, Hope \& Kelly (1983), Kahneman, Slovic \& Tversky (1982). Estos últimos han señalado la utilización que las personas hacen de heurísticos, entendidos como procedimientos tendientes a reducir la complejidad de la información disponible en situaciones reales para la cual la mente humana encuentra limitaciones. Especialmente destacado es el heurístico de representatividad que consiste en adjudicar mayor o menor probabilidad a un suceso según sea representativo o no de la población de origen, desestimando otras características de la muestra tales como el tamaño e indirectamente la variabilidad del muestreo. Es decir, existe una tendencia a sobreestimar la probabilidad de un resultado basado en una muestra pequeña (Tversky \& Kahneman, 1971). De esta forma se otorga a un número limitado de ensayos la capacidad de reproducir todas las características de la población, lo cual puede llevar a la realización de juicios probabilísticos erróneos, aun en personas formadas en teoría de probabilidades.

Díaz Godino, Batanero \& Cañizares (1991) se han preguntado desde el ámbito educativo cómo controlar y minimizar los sesgos en el aprendizaje de conceptos probabilísticos y tomaron las recomendaciones que sugiere Shaughnessy (1982), que podrían sintetizarse en: presentar a los alumnos situaciones problemáticas que involucren la Probabilidad y la Estadística a través de la recolección y simulación de datos, cuestionando creencias personales a partir de la evidencia experimental y permitiendo la toma de decisiones en situaciones de incertidumbre con una base racional. Así como también, señalar la existencia de sesgos en el pensamiento, la utilización errónea de conceptos estadísticos, paradojas aparentes, realizando investigaciones clínicas sobre los alumnos para estudiar modos de pensar acerca de situaciones aleatorias. 
El objetivo de este trabajo fue evaluar la habilidad para analizar secuencias aleatorias en las que se debió tener en cuenta la estabilidad de las frecuencias relativas. Se estudia una de las diferentes manifestaciones del heurístico de representatividad que lleva a insensibilizar las estimaciones respecto del tamaño de la muestra de donde se extrae la evidencia, haciéndose un uso inadecuado de la ley de los grandes números al aplicarla a un número reducido de ensayos, error conocido como ley de los pequeños números. También se estudia la estimación de probabilidades para la que hay que tener en cuenta una variable contadora y su distribución probabilística, generada a partir de una secuencia aleatoria dicotómica. La falta de una consideración detenida acerca de las situaciones aleatorias presentadas, de sencilla enunciación, pueden conducir a la sobreestimación de la potencia de los métodos estadísticos empleados, subestimación de la amplitud de los intervalos de confianza, así como a expectativas injustificadas en la replicabilidad de los experimentos basados en muestras pequeñas (Serrano et al., 1998).

\section{Método}

\section{Diseño de Actividades}

Se diseñaron dos reactivos que permitieron indagar estimaciones de probabilidad en situaciones en las que interviene el azar. En el primero, denominado Reactivo del Sesgo de Proporciones (SP) (adaptado de Kahneman \& Tversky, 1972), dicha indagación se efectuó al comparar proporciones de dos muestras de diferente tamaño y en las que se debió tener en cuenta la ley de los grandes números. En el segundo, denominado Reactivo de Estimación de Probabilidad (EP), esta estimación se basó en las probabilidades (todas iguales entre sí) que aportan las distintas secuencias de nacimientos a los valores de la variable que registra la cantidad de nacimientos de un mismo sexo generada a partir de una secuencia aleatoria. Se utilizaron planteos de situaciones secuenciales azarosas, buscando construir categorizaciones de respuestas.

A continuación se muestran los reactivos diseñados.

Reactivo del Sesgo de Proporciones (SP)

Se registran los nacimientos diarios en dos centros de salud, A y B. El centro A tiene menor infraestructura que el B. Teniendo en cuenta que aproximadamente el $50 \%$ de los bebés que nacen son varones, ¿qué es más probable que ocurra entre estas dos opciones a acontecer en un mismo día?

a) que nazcan 8 o más varones de 10 bebés nacidos en el centro $A$.

b) que nazcan 80 o más varones de 100 bebés nacidos en el centro $B$.

\section{Reactivo de Estimación de Probabilidad (EP)}

Se sabe que aproximadamente el $50 \%$ de los bebés recién nacidos son varones. En un Hospital se han registrado 1000 nacimientos de los cuales 550 fueron varones. En 1000 nacimientos futuros, ¿qué pensás que es más probable que ocurra?

a) que nazcan por lo menos 550 varones.

b) que nazcan menos de 550 varones.

\section{Participantes}

Se administraron los dos reactivos a 218 jóvenes ingresantes a la Universidad de Buenos Aires, sin conocimientos previos acerca de las teorías de la probabilidad, provenientes de colegios públicos y privados de nivel socioeconómico medio. La muestra fue seleccionada por accesibilidad. 


\section{Resultados}

En base a las nociones teóricas mencionadas en el diseño de actividades se categorizaron las respuestas brindadas por los alumnos para ambos reactivos.

A continuación se define la categorización para el Reactivo SP y se presenta la distribución correspondiente en porcentaje para la muestra $(\mathrm{n}=218)$ en la Tabla 1.

1) Correcto: respuestas que distinguieron que los apartamientos de la cantidad de varones en $n$ nacimientos respecto del valor esperado es más fácil de encontrar en series de menor tamaño, razón por la cual se inclinaron por la opción a).

2) Igual probabilidad: respuestas que, desestimando el tamaño de la muestra, otorgaron, erróneamente, igual probabilidad a ambas opciones, justificándose en la igualdad de proporciones (8/10 es igual a 80/100).

3) Más probable opción b) (muestra de mayor tamaño): respuestas que optan por la opción b) confiriéndole mayor confianza dado que presenta mayor tamaño muestral, haciendo referencia a que de esta forma disminuye el error de estimación, cuestión, esta última, que no es incorrecta pero que no responde a la pregunta presentada.

4) Mas probable opción a) (muestra de menor tamaño): respuestas que optaron por el suceso de la opción a) que, a pesar de ser la opción correcta, fue fundamentada incorrectamente. Se hace referencia a que al tratarse de una muestra de menor tamaño, era más fácil de ser contrastada mediante réplicas de la experiencia, cuestión que se desvía del tema consultado.

5) No interpreta: respuestas que no interpretaron el reactivo presentado dando argumentos totalmente alejados de las cuestiones consultadas.

6) No contesta: Ausencia de respuestas.

\section{Reactivo SP}

\begin{tabular}{|c|c|}
\hline Categoría & $\begin{array}{c}\text { Porcentaje } \\
\mathrm{n}=218\end{array}$ \\
\hline 1.- Correcto & 28 \\
\hline 2.- Igual probabilidad & 42 \\
\hline $\begin{array}{c}\text { 3.- Más probable opción b) (muestra de mayor } \\
\text { tamaño): }\end{array}$ & 20 \\
\hline $\begin{array}{c}\text { 4.- Mas probable opción a) (muestra de menor } \\
\text { tamaño) }\end{array}$ & 3 \\
\hline 5.- No interpreta & 3 \\
\hline 6.- No contesta & 4 \\
\hline Total & 100 \\
\hline
\end{tabular}

\section{Tabla 1}

Para la misma muestra $(n=218)$ se define la siguiente categorización para el Reactivo EP y su distribución en porcentaje en la Tabla 2.

1) Correcto: respuestas que optaron por la opción b) dado que incluye mayor cantidad de configuraciones y, por lo tanto, aportan mayor probabilidad a la cantidad de nacimientos de varones mencionada en dicha opción. 
2) Intuición correcta con justificación menos adecuada: Al igual que en la categoría anterior los participantes optaron por la opción b), dando argumentos que apelan a la compensación de resultados (ya que en el enunciado del reactivo se mencionan más varones que mujeres) al estilo de la falacia del jugador. Este efecto se detectó en trabajos previos (Attorresi, García Díaz \& Pralong, 2006, 2007).

3) Igual Probabilidad: respuestas que otorgan igual probabilidad a las dos opciones planteadas, a) y b), apelando a la igualdad de posibilidades en cuanto al sexo en un determinado nacimiento. Estas respuestas son erróneas ya que confunden la probabilidad de ocurrencia de un sexo determinado en un nacimiento, situación dicotómica y equiprobable, con la probabilidad de ocurrencia de cierta cantidad de bebés de un sexo determinado en una secuencia aleatoria de n nacimientos, variable esta última, cuyos valores no son equiprobables, siendo la opción b) la que reúne los valores de dicha variable que tienen mayor probabilidad.

4) No optan por ninguna opción: respuestas que no optan por ninguna opción, dando argumentos de hechos aislados como la similar chance para ambos sexos en un nacimiento, o bien la existencia de condiciones ambientales que determinan el nacimiento o apelan a una imprevisibilidad absoluta del azar.

5) Mas probable opción a): Reúne a las respuestas que optaron erróneamente por elegir la opción a), probablemente por conferirle a la realización particular del experimento dado en el enunciado "nacieron 550 varones" (y por lo tanto 450 mujeres) el valor de una evidencia empírica determinante para la asignación de probabilidad en ese sentido, es decir, otorgar mayor probabilidad a las series con mayor cantidad de varones.

6) No interpreta: respuestas que no interpretaron el reactivo presentado dando argumentos totalmente alejados de las cuestiones consultadas.

7) No contesta: Ausencia de respuestas.

\section{Reactivo EP}

\begin{tabular}{|c|c|}
\hline Categoría & $\begin{array}{c}\text { Porcentaje } \\
\mathrm{n}=218\end{array}$ \\
\hline 1.- Correcto & 14 \\
\hline 2.- Intuición correcta con justificación menos adecuada & 6 \\
\hline 3.- Igual probabilidad & 28 \\
\hline 4.- No optan por ninguna opción & 26 \\
\hline 5.- Más probable opción a) & 15 \\
\hline 6.- No interpreta & 4 \\
\hline 7.- No contesta & 7 \\
\hline Total & 100 \\
\hline
\end{tabular}

Tabla 2

Asociación entre los reactivos

Se reunieron las categorías para cada reactivo en dos grupos. Uno contenía las argumentaciones esencialmente correctas que para el reactivo SP corresponde a la categoría 1, 
mientras que para el EP corresponde a las categorías 1 y 2. El otro contenía argumentos incorrectos en distinto grado, correspondiendo a las categorías 2 a 5 del reactivo SP y a las categorías 3 a 6 del EP.

Se efectuó un análisis conjunto de las respuestas dicotomizadas en correctas e incorrectas, descartándose los protocolos en los que no hubo respuesta para alguna de dichas situaciones de modo que el tamaño de muestra se restringió a 199 casos. Como se muestra en la tabla 3, con un nivel de significación del 5\%, se encontró asociación entre los reactivos.

\begin{tabular}{|c|c|c|c|}
\hline \multirow{2}{*}{ Reactivo SP } & \multicolumn{2}{|c|}{ Reactivo EP } & \multirow{2}{*}{ Total } \\
\hline & Correcta & Incorrecta & \\
\hline Correcta & \multirow{4}{*}{$\begin{array}{c}18 \\
12.16 \\
2.80\end{array}$} & \multirow{4}{*}{$\begin{array}{c}37 \\
42.84 \\
0.80\end{array}$} & \\
\hline Frec. Observadas & & & 55 \\
\hline Frec. Esperadas & & & \\
\hline Celda chi-cuadrado & & & \\
\hline Incorrecta & \multirow{4}{*}{$\begin{array}{c}26 \\
31.84 \\
1.07\end{array}$} & \multirow{4}{*}{$\begin{array}{c}118 \\
112.16 \\
0.30\end{array}$} & \\
\hline Frec. Observadas & & & 144 \\
\hline Frec. Esperadas & & & \\
\hline Celda chi-cuadrado & & & \\
\hline Total & 44 & 155 & 199 \\
\hline \multicolumn{4}{|c|}{ Nota. $\mathrm{Chi}=4.97 ; \mathrm{p}=0.026$} \\
\hline
\end{tabular}

\section{Tabla 3}

El principal aporte a la asociación fue brindado por las respuestas correctas en ambos reactivos. La proporción de justificaciones correctas en el reactivo SP e incorrectas en el EP fue ligeramente superior que a la inversa, lo cual puede dar cuenta de que la situación enunciada en el reactivo EP es algo más compleja que en el SP. En este mismo sentido, se advierte que el porcentaje de respuestas que no interpretaron y/o no contestaron las situaciones planteadas fue mayor en el segundo que en el primer reactivo ( $11 \%$ vs. $7 \%)$ (ver Tablas 1 y 2$)$.

\section{Discusión}

Para el reactivo SP se encontró que algo menos de un tercio de los participantes dieron argumentos correctos ante la situación presentada. El resto de las categorías reunieron argumentaciones incorrectas aunque en distinto grado. El error más frecuente, que superó en porcentaje al de las respuestas correctas, fue la asignación de igual probabilidad a las dos opciones del reactivo presentado. De este modo se prestó atención a la igualdad de proporciones de las muestras y se desatendió la cuestión del tamaño de las mismas, idea central vinculada a la estabilidad de las frecuencias relativas y que por lo tanto condujo a respuestas erróneas. Otra categoría que resultó significativa en porcentaje fue la que reunió a las preferencias por la opción b) con justificaciones basadas en una idea correcta de que a mayor tamaño de muestra menor error de estimación. Esta justificación no condujo a la cuestión central de que el alejamiento de los valores de la variable aleatoria cantidad de varones en $n$ nacimientos respecto de su valor esperado es más probable en muestras de menor tamaño y que por lo tanto llevó erróneamente a elegir la opción b).

Para el reactivo EP las respuestas correctas, desde las más adecuadas en cuanto a su justificación a las menos, con argumentos más intuitivos, representan la quinta parte del total. El resto de las categorías resultaron incorrectas. En este segundo grupo la mayoría nuevamente otorgó igual probabilidad a las dos opciones del reactivo presentado. No se distinguió que el efecto de la repetición de los nacimientos daba origen a una variable contadora a la que se hace mención en el enunciado del reactivo, que registraba la cantidad de nacimientos de bebés de un determinado sexo 
(en este trabajo varones) cuyos valores no son equiprobables. Este hecho no debería confundirse con que cada serie de n nacimientos, tenga la misma probabilidad ya que se compone de $\mathrm{n}$ ensayos aleatorios tipo Bernoulli con probabilidad de éxito 0.5 y que a distintos números de nacimientos de un determinado sexo les corresponden distintas cantidades de estas series o configuraciones, aportando de este modo distinta probabilidad. El no tener en cuenta estas consideraciones puede llevar a resultados erróneos como el de no distinguir que los valores de la variable incluidos en la opción b) tienen mayor probabilidad que los incluidos en la a) y por lo tanto dichas opciones no son equiprobables. Parte de las respuestas reunidas en la categoría 4 apelaron también a argumentos de equiprobabilidad y a otras consideraciones que hicieron que los participantes no optaran por ninguna de las opciones. Este sesgo de equiprobabilidad que aparece en las categorías 3 y 4 supera la mitad de las justificaciones para la situación planteada. Cabe mencionar que las nociones que fundamentan la respuesta correcta exigen la consideración de dos variables distintas. Una de ellas es una variable que responde al modelo de Bernoulli, la que registra el sexo en un nacimiento, cuyos dos valores (éxito o fracaso) son equiprobables en este caso, mientras que la otra es una variable que corresponde al modelo binomial que contabiliza la cantidad de éxitos ocurridos en n realizaciones independientes de la variable Bernoulli, cuyos valores de 0 a n no son equiprobables. Las dificultades presentadas por los participantes en este estudio para efectuar esta distinción aparecen con bastante regularidad en los cursos básicos de Probabilidad y Estadística dictados por los autores de este trabajo en su tarea como docentes cuando se abordan estos temas. Lo sugestivo de este hecho es que en este último caso, se trata de estudiantes que se hallan cursando su primer año de Universidad y que están recibiendo una instrucción paralela sobre estos temas. Es posible entonces observar que, si bien las situaciones presentadas en este trabajo son de sencilla formulación en el lenguaje natural, es decir, las personas consultadas entienden el enunciado del problema presentado, no ocurre lo mismo con los conceptos e ideas que son necesarios para su adecuada resolución. Ésta última, demanda recursos cognitivos que exceden el marco de la intuición y que requieren de una consideración teórica y reflexiva particular sobre el tema que no necesariamente es alcanzada con una instrucción básica en probabilidades.

La asociación encontrada entre las respuestas de ambos reactivos, dicotomizadas entre correctas e incorrectas, es debida principalmente a los participantes que tuvieron mejor desempeño.

Los resultados encontrados permiten rescatar las recomendaciones de Shaughnessy (1982) quien, además de lo mencionado al final de la introducción de este trabajo, propone estudiar en los razonamientos de los estudiantes la existencia de sesgos en el pensamiento, la utilización errónea de conceptos estadísticos y paradojas aparentes. Con este fin sugiere realizar investigaciones mediante entrevistas clínicas para estudiar los modos de pensar las situaciones aleatorias mencionadas. 


\section{Referencias}

Attorresi H., García Díaz A. \& Pralong H. (2006). Identificación de la Falacia del Jugador en una situación típica de juego de azar. Memorias de las XIII Jornadas de Investigación de la Facultad de Psicología. Universidad de Buenos Aires, 13-2,184-186.

Attorresi H., García Díaz A. \& Pralong H. (2007). Sesgos en la Comprensión de Dos Situaciones Típicas de Incertidumbre y de Azar. Perspectivas en Psicología, 4-1, 28-37.

Barragués, J., Guisasola, J. \& Morais, A. (2005). Concepciones de los estudiantes de Primer Ciclo de Universidad sobre estimación de la probabilidad. Educación Matemática, 17-1, 55-85.

Batanero, C. (2000). ¿Hacia dónde va la educación estadística?. Blaix, 15, 2-13.

Borovcnik, M. \& Bentz, H. (1991). Empirical Research in Understanding Probability. En R. Kapadia \& M. Borovcnik (eds.), Chance Encounters: Probability in Education, pp. 73-105. Países Bajos: Kluwer Academic Publisher.

Borovenik, M. \& Peard, R. (1996). Probability en A. Bishop, K. Clements, C. Keitel, J. Kilpatrick, \& C. Laborde (eds.), International Handbook of mathematics Education, pp. 239-287. Países Bajos: Kluwer Academic Publishers.

Cardeñoso, J. (2001). Las creencias y conocimientos de los profesores de primaria andaluces sobre la matemática escolar. Modelización de concepciones sobre la aleatoriedad y probabilidad. Tesis Doctoral. Cádiz: Servicio de Publicaciones de la Universidad de Cádiz.

Díaz, C. (2003). Heurísticas y sesgos en el razonamiento probabilístico. Implicaciones para la enseñanza de la Estadística. 27 Congreso Nacional de Estadística e Investigación Operativa. Leida.

Díaz Godino, J., Batanero Bernabeu, C. \& Cañizares Castellano, J. (1991). Azar y Probabilidad, Matemáticas: cultura y aprendizaje, 27. Madrid: Síntesis.

Fischbein, E. (1975). The intuitive sources of probability thinking in children. Dordrecht: Reidel.

Guisasola, J. \& Barragués, J. (2002). Heurísticas y sesgos de los estudiantes de primer ciclo de universidad en la resolución de problemas de probabilidad. Enseñanza de las Ciencias, 20-2, 285-302.

Hope, J. \& Kelly, I. (1983). Common difficulties with probabilistic reasoning. Mathematics Teacher, $76,565-570$.

Kahneman, D. \& Tversky, A. (1972). Subjective probability: a judgement of representativeness. Cognitive Psychology, 3, 430-454.

Kahneman, D., Slovic, P. \& Tversky, A. (1982). Judgment under uncertainty: heuruistics and biases. Cambridge: University Press.

Konold, C. (1989). Informal Conceptions of probability. Cognition and Instruction, 6, 59-98.

Konold, C. (1991). Understanding Students' Beliefs about Probability. En E. von Glasesfeld (ed.), Radical constructivism in Mathematics Educations, pp. 139-156. Dordrecht: Kluwer.

Lecoutre, M. (1985). Effect d' informations de nature combinatoire et de nature frécuentielle sur le judgements probabilistes. Recherches en Didactique des Mathématiques, 6, pp. 193-213.

Lecoutre, M. (1992). Cognitive Models and Problem Spaces in Purely Random Situations Educational Studies in Mathematics, 19, pp. 357-368.

Lecoutre, M. \& Cordier, J. (1990). Effet du mode de présentation d'un problème aleatoire sur les modèles développés par les élèves. Bulletin de l'APMEP, 372, 9-22. 
Piaget, J. \& Inhelder, B. (1974). La genèse de l'idèe de hasard chez l'enfant. Paris: Press Universitaires de France.

Serradó, A., Cardeñoso, J. \& Azcárate, P. (2005). Los obstáculos en el aprendizaje del conocimiento probabilístico: su incidencia desde los libros de texto. Statistics Education Research Journal, $4-2,59-81$.

Serrano, L., Batanero C., Ortiz J. \& Cañizares J. (1998). Heurísticos y sesgos en el razonamiento probabilístico de los estudiantes de secundaria. Educación Matemática, 10 -1, 7-25.

Shaughnessy, J. (1982). Misconceptions of probability, systematics and otherwise; teaching probability and statistics so as to overcome some miscoconceptions. Proceedings of ICOTS. 2, 784-801. Universidad de Sheffield.

Tvesky, A. \& Kahneman, D. (1971). The belief in the law of small numbers. Psychological Bulletin, 76, 105-110. 